INT. J. SYSTEMS SCI., I984, vOL. 15, No. 5, 513-524

\title{
Time-optimal control policies for cascaded production-inventory systems with control and state constraints $\dagger$
}

\author{
J. WARSC'HAT $\ddagger$ and H. J. IVUNDERLICH $\S$
}

In this paper time-optimal control policies are derived for models of productioninventory systems consisting of a cascade of basic production-inventory systems with control and state constraints. The analytic solution is due to a decoupling of the complete system into its subsystems by a recursive definition of the cascaded system. It is shown that there is at least one bang-bang controlled subsystem. For the ' other' subsystems singular control policies are obtained. Introducing a pseudobang-bang control for these systems it is demonstrated that by strengthening the constraints there is a continuous transition from a singular to a bang-bang control.

\section{Introduction}

In a recent paper (Bradshaw and Erol 1980) sub-optimal control policies were derived for a class of linear time-inyariant models of production-inventory systems consisting of a cascade of $m$ basic production-inventory systems with bounded inputs. The control policies were obtained by the transformation of the continuous-time model into a discrete-time model and a subsequent application of a dead-beat control policy.

In this paper it is shown that the control policies can be derived by an analytic solution defining the cascaded system in a recursive manner. Often it is necessary to impose constraints on the state variables too. Concerning the production-inventory systems this case could arise if the inventories must be regarded with natural limits or if an overtension of the system must be avoided which leads to a restriction of the production rate. In this paper we consider the latter case.

The state constrained system is treated by the application of the theory developed by Jacobson et al. (1971), Hamilton (1972) and Maurer (1977). The theory is illustrated by the presentation of the state and control variables of two production-inventory systems in cascade.

\section{Systems with linear control. Necessary conditions}

Before treating the cascaded production-inventory system a short presentation of necessary conditions is given concerning state constrained systems. The state of the problem and the necessary conditions follow Maurer (1977). Thereby a modifcation is introduced by formulating the minimum principle for multiple input and multiple state constraint. Moreover, as will be clear in the sequel, only scalar input and scalar constraint will be necessary due to

Received 6 June 1983.

† This research was supported by the Stiftung Volkswagenwerk.

$\ddagger$ Fraunhofer-Institut für Arbeitswirtschaft und Organisation, Silberburg-str. 119 A, D-7000 Stuttgart 1, West Germany.

§ Institut für Informatik IV, Universität Karlsruhe, Zirkel 2, D-7500 Karlsruhe 1, West Germany. 
the decoupling of the system into its subsystems. So Maurer's theory holds for the following problem: determine a piecewise continuous control $u(t), t \in[0,1]$, which minimizes the functional

$$
J(u):=F(x(1))
$$

subject to

$$
\begin{aligned}
\dot{x}(t) & =f(x(t), u(t)):=f_{1}(x(t))+f_{2}(x(t)) u(t) \\
x(0) & =x_{0}, \quad Q(x(1))=0 \\
u(t) \epsilon I^{\cdot}: & =\left\{u \mid \zeta_{j} \leqq u_{j} \leqq \xi_{j}, \quad j=1, \ldots, m\right\} \subset \mathbb{R}^{m} \\
S_{i}(x(t)) & \leqq 0, \quad i=1, \ldots, h
\end{aligned}
$$

with the state vector $x(t) \in \mathbb{R}^{n}$, the control vector $u(t) \in \mathbb{R}^{m}$, the functions $F: \mathbb{R}^{n} \rightarrow \mathbb{R}, Q: \mathbb{R}^{n} \rightarrow \mathbb{R}^{k}, k \leqq n, f_{1}: \mathbb{R}^{n} \rightarrow \mathbb{R}^{n}, f_{2}: \mathbb{R}^{n} \rightarrow \mathbb{R}^{n \cdot m}$ and $S: \mathbb{R}^{n} \rightarrow \mathbb{R}^{h}$. $h \leqq m$, which are assumed to be sufficiently many times continuously differentiable.

\section{Definition}

The interval

$$
I:=\left[t_{1}, t_{2}\right] \subset[0,1]
$$

is called the boundary arc and $t_{1}$ and $t_{2}$ are called entry-and exit-time, respectively, of the trajectory $x(t)$ concerning $S_{i}$, if there exists a value $\epsilon>0$ with $S_{i}(x(t)) \neq 0$ for $t_{1}-\epsilon \leqq t<t_{1}$ and for $t_{2}<t \leqq t_{2}+\epsilon$ and $S_{i}(x(t))=0$ for $t \in I$. $I$ is called interior arc if $I$ is disjoint with any boundary arc.

\section{Definition}

For each component $S_{i}(x)$ of the constraint $S(x), i=1, \ldots, h$, let the order $p_{i}$ be the smallest integer, such that the $p_{i}$ th time derivative of $S(x(t))$ contains $u(t)$ explicitly.

For each optimal solution $x(t), u(t)$ of the problem (1) to (5) there exists an adjoint vector $\lambda(t) \in \mathbf{R}^{n}$, a real vector $\sigma \in \mathbb{R}^{k}$ and a measurable function $\eta:[0,1] \rightarrow R^{h}$ with $\eta^{\mathrm{T}}(t) S(x(t)) \equiv 0$, where $\mathrm{T}$ denotes the transpose, $t \in[0,1]$, satisfying (7) to (11)

$$
\begin{aligned}
\lambda^{\mathrm{T}} & =-\lambda^{\mathrm{T}} f_{1 x}(x)-\lambda^{\mathrm{T}} f_{2 x}(x) u-\eta^{\mathrm{T}} S_{x}(x) \\
\lambda^{\mathrm{T}}(1) & =F_{x}(x(1))+\sigma^{\mathrm{T}} Q_{x}(x(1))
\end{aligned}
$$

This relation holds for problems fulfilling the Slater condition (see Girsanov 1972). The stated problem is assumed to satisfy this condition. Setting

$$
\nu(l):=\eta\left(t^{+}\right)-\eta\left(t^{-}\right)
$$

hence

$$
\lambda^{\mathrm{T}}\left(t^{+}\right)=\lambda^{\mathrm{T}}\left(t^{-}\right)-v^{\mathrm{T}}(t) S_{x}(x(t)), \quad v_{i}(t) \geqslant 0
$$

Discontinuities $\left(v_{i}(t)>0\right)$ can only occur at entry- and at exit-points for $S_{i}$. With the hamiltonian

$$
H(x(t), u(t), \lambda(t)):=\lambda^{\mathrm{T}}(t) f_{1}(x(t))+\lambda^{\mathrm{T}}(t) f_{2}(x(t)) u(t)+\eta^{\mathrm{T}}(t) S(x(t))
$$


the minimum principle takes the form

$$
H(x(t), u(t), \lambda(t))=\min _{u \in I^{-}} H(x(t), u, \lambda(t))
$$

Autonomous systems with a free end time $T$ may be transformed into a problem (1) to (5) by introducing an additional state variable and a subsequent substitution. At last we get

$$
\dot{x}(t)=T f_{1}(x(t))+T f_{2}(x(t)) u(t), \quad t \in[0,1]
$$

and as further condition

$$
H(x(1), u(1), \lambda(1))=-1
$$

The switching function for the control $u$ is

$$
\phi^{\mathrm{T}}(t):=\lambda^{\mathrm{T}}(t) f_{2}(x(t))
$$

For each component $\phi_{j}{ }^{\mathrm{T}}(t) \neq 0$ the minimum principle yields to the bang-bang control

$$
u_{j}(t)= \begin{cases}\zeta_{j} & \text { if } \phi_{j}(t)>0 \\ \xi_{j} & \text { if } \phi_{j}(t)<0\end{cases}
$$

\section{Cascaded production-inventory systems}

\subsection{State equations}

Any cascaded production-inventory system, consisting of $m$ subsystems, can be represented by the block diagram shown in Fig. 1.

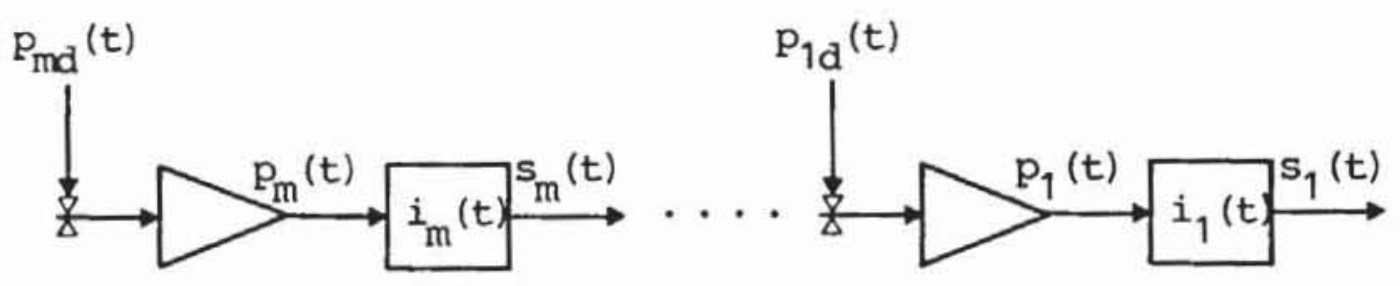

Figure 1.

The variables associated with the $j$ th subsystem $(j=1, \ldots, m)$ have the following meanings : $p_{j d}(t)$ is the desired production rate; $p_{j}(t)$ is the actual production rate $; i_{j}(t)$ is the actual inventory level ; $s_{j}(t)$ is the shipping rate.

If it is assumed that the production process in each subsystem can be represented by the first order exponential delay with the time constant $1 / \alpha_{j}$ $(j=1,2, \ldots, m)$, then the governing equations for the subsystems are

$$
\left.\begin{array}{c}
i_{j}(t)=p_{j}(t)-s_{j}(t) \\
\dot{p}_{j}(t)=\alpha_{j}\left(p_{j d}(t)-p_{j}(t)\right)
\end{array}\right\} j=1, \ldots, m
$$

It is assumed that

$$
s_{j+1}(t)=p_{j d}(t), \quad j=1, \ldots, m
$$


The aim is to find a control policy $p_{j d}(t)$ which transfers the system (16) to (18) from a given initial equilibrium state

$$
\left.\begin{array}{c}
i_{m}(0), i_{m-1}(0), \ldots, i_{1}(0) \\
p_{m d}(0)=p_{m}(0)=s_{m}(0)=\ldots=p_{1 d}(0)=p_{1}(0)=s_{1}(0)=s_{10}
\end{array}\right\}
$$

to a given final equilibrium state

$$
\left.\begin{array}{c}
i_{m}(T), i_{m-1}(T), \ldots, i_{1}(T) \\
p_{m d}(T)=p_{m}(T)=s_{m}(T)=\ldots=p_{1 d}(T)=p_{1}(T)=s_{1}(T)=s_{1 T}
\end{array}\right\}
$$

in minimum time $T$, subject to the constraints

$$
s_{1 T}-\rho \leqq p_{j d}(t) \leqq 8_{1 T}+\rho, \quad j=1, \ldots, m, \quad \rho \in \mathbf{R}^{+}
$$

Throughout the paper we assume $\rho=1$. With

$$
\left.\begin{array}{r}
u_{j}(t)=p_{j d}(t)-p_{j d}(T) \\
x_{2 j-1}(t)=i_{j}(t)-i_{j}(T) \\
x_{z(}(t)=p_{j}(t)-p_{j}(T)
\end{array}\right\} \quad j=1, \ldots, m
$$

and the transformation (12) the following state equations are obtained

$$
\dot{x}(t)=T A x(t)+T B u(t)
$$

where

$$
x^{\mathrm{T}}=\left[x_{1}, \ldots, x_{2 m}\right], \quad u^{\mathrm{T}}=\left[u_{1}, \ldots, u_{m}\right]
$$

and

$$
\left.\begin{array}{rl}
A & =\left[\begin{array}{ccccccc}
0 & 1 & 0 & 0 & \ldots & 0 & 0 \\
0 & -\alpha_{1} & 0 & 0 & \ldots & 0 & 0 \\
0 & 0 & 0 & 1 & \ldots & 0 & 0 \\
0 & 0 & 0 & -\alpha_{2} & \ldots & 0 & 0 \\
0 & 0 & 0 & 0 & \ldots & 0 & 1 \\
0 & 0 & 0 & 0 & \ldots & 0 & -\alpha_{m}
\end{array}\right] \\
B & =\left[\begin{array}{cccccc}
0 & 0 & 0 & \ldots & 0 & 0 \\
\alpha_{1} & 0 & 0 & \ldots & 0 & 0 \\
-1 & 0 & 0 & \ldots & 0 & 0 \\
0 & \alpha_{2} & 0 & \ldots & 0 & 0 \\
0 & 0 & 0 & \ldots & -1 & 0 \\
0 & 0 & 0 & \ldots & 0 & \alpha_{m}
\end{array}\right]
\end{array}\right\}
$$


From (19), (20) and (22) we get the initial conditions

$$
x_{2 j-1}(0)=0, \quad x_{2 j}(0)=s_{10}-s_{1 T}
$$

\subsection{Control policies for a submodel}

First let us consider an unbounded subproblem of the cascaded system (23) to (26).

(SP) We seek a control $u(t) \in \mathbb{R}, t \in[0,1]$ with $|u(t)| \leqq 1$ and $T$ minimal, such that the trajectory $x(t) \in \mathbb{R}^{2}$ is driven from $x^{\mathrm{T}}(0)=[0, a]$ to $x^{\mathrm{T}}(1)=[b, 0]$ according to the equation

$$
\dot{x}(t)=T\left[\begin{array}{cc}
0 & 1 \\
0 & -\alpha
\end{array}\right] x(t)+T\left[\begin{array}{l}
0 \\
\alpha
\end{array}\right] u(t), \quad t \in[0,1], \quad \alpha>0
$$

The hamiltonian is

$$
H(x(t), u(t), \lambda(t))=\left(\lambda_{1}(t)-\alpha \lambda_{2}(t)\right) x_{2}(t)+\alpha \lambda_{2}(t) u(t)
$$

The adjoint equation becomes

$$
\lambda=-T\left[\begin{array}{cc}
0 & 0 \\
1 & -\alpha
\end{array}\right] \lambda .
$$

It follows from (9) that $\lambda$ is continuous and therefore one obtains

$$
\begin{aligned}
& \lambda_{1}(t)=c \\
& \lambda_{2}(t)=d \exp (T \alpha t)+c / \alpha
\end{aligned}
$$

with some integration constants $c$ and $d$.

The case $\lambda_{2} \equiv 0$ is excluded, since this would be a contradiction to (13). Furthermore $\lambda_{2}$ is a monotonous function with at most one zero. The switching function is $\phi(t) \geqslant \alpha \lambda_{2}(t)$ and therefore the system (27) turns out to be bang-bang controlled

$$
u(t)=\left\{\begin{aligned}
1 & \text { if } \lambda_{2}(t)<0 \\
-1 & \text { if } \lambda_{2}(t)>0
\end{aligned}\right.
$$

Transforming (27) into integral equations with respect to the initial conditions we obtain

$$
x_{1}(t)=T \int_{0}^{t} x_{2}(\tau) d \tau
$$

and

$$
x_{2}(t)=\exp (-T \alpha t) T \alpha \int_{0}^{t} u(\tau) \exp (T \alpha \tau) d \tau+a \exp (-T \alpha t)
$$

From (34) we get with the final conditions of (SP)

$$
\int_{0}^{1} u(\tau) \exp (T \alpha \tau) d \tau=-a / T \alpha
$$


Equations (33) and (34) and the final conditions yield in accordance with the well known theorem of Fubini

$$
\begin{aligned}
b & =T\left(\int_{0}^{1} \exp (-T \alpha t) T \alpha \int_{0}^{t} u(\tau) \exp (T \alpha \tau) d \tau d t-a \frac{\exp \left(-T_{\alpha}\right)-1}{T \alpha}\right) \\
& =T\left(\int_{0}^{1} \int_{\tau}^{1} \exp \left(-T_{\alpha} t\right) T \alpha u(\tau) \exp (T \alpha \tau) d t d \tau-a \frac{\exp \left(-T_{\alpha}\right)-1}{T \alpha}\right) \\
& =T\left(\int_{0}^{1} u(\tau) d \tau-\exp (-T \alpha) \int_{0}^{1} u(\tau) \exp \left(T_{\alpha \tau}\right) d \tau-a \frac{\exp \left(-T_{\alpha}\right)-1}{T \alpha}\right)
\end{aligned}
$$

Together with (35) we now obtain

$$
\int_{0}^{1} u(\tau) d \tau=\frac{b}{T}-\frac{a}{T \alpha}
$$

Because of (32) $u$ is a bang-bang control, i.e. there exists a $t_{s} \in[0,1]$ with

$$
u(t)=\left\{\begin{array}{rr}
1, & 0 \leqq t \leqq t_{s} \\
-1, & t_{s}<t \leqq 1
\end{array}\right.
$$

or

$$
u(t)=\left\{\begin{array}{rr}
-1, & 0 \leqq t \leqq t_{s} \\
1, & t_{s}<t \leqq 1
\end{array}\right.
$$

Integrating (33) and (34) with (37) and (37') respectively the following two systems of equations turn out

$$
\left.\begin{array}{rl}
2 \exp \left(T \alpha t_{s}\right)-\exp (T \alpha)-1 & =-a \\
2 t_{s}-1 & =\frac{b}{T}-\frac{a}{T \alpha}
\end{array}\right\}
$$

From (38) and $\left(38^{\prime}\right)$ respectively we take the solution with minimal time $T$ as the optimal solution.

Now let us consider the bounded subproblem.

(SPB) This consists of the (SP) with the additional state constraint

$$
S(x(t)):=x_{2}(t)-\beta \leqq 0, \quad t \in[0,1]
$$

The time derivative of $S(x(t))$ is

$$
S^{1}(x(t))=T[0,-\alpha] x(t)+T \alpha u=T \alpha x_{2}(t)+T \alpha u(t)
$$

Thus $p=1$ is the order of the constraint and if there exists any boundary arc $I$ then $S^{1}(x(t))=0$ for $t \in I$ is valid. This yields to the boundary control :

$$
u(t)=x_{2}(t)=\beta
$$


Maurer (1977) has shown that the adjoint vector function is continuous at entry - and exit-points for constraints of order $p=1$. If the constraint is active, i.e. the solution of the (SP) violates (39), the switching function vanishes on the boundary arc : $\phi(t)=\lambda_{2}(t) \equiv 0$. Consequently the equation (7) takes the form

$$
\lambda=\left\{\begin{array}{cc}
-T\left[\begin{array}{cc}
0 & 0 \\
1 & -\alpha
\end{array}\right] \lambda & \text { at an interior arc } \\
0 & \text { at a boundary arc }
\end{array}\right.
$$

This yields to the monotonous function $\lambda$ as for the (SP) and hence the structure of the optimal trajectory is 'interior arc '- 'boundary arc '- 'interior arc'. Thus the optimal control becomes

In (34) we set $x\left(t_{1}\right)=\beta$ and get

$$
u(t)=\left\{\begin{array}{rr}
1, & 0 \leqq t<t_{1} \\
\beta, & t_{1} \leqq t<t_{2} \\
-1, & t_{2} \leqq t \leqq 1
\end{array}\right.
$$

$$
t_{1}=\frac{\ln \frac{a-1}{\beta-1}}{T_{\alpha}}
$$

Applying (43) to (35) and (36) the following equations hold

and

$$
t_{1}+\beta\left(t_{2}-t_{1}\right)+t_{2}-1=\frac{b}{T}-\frac{a}{T_{\alpha}}
$$

$$
\exp \left(T \alpha t_{1}\right)-1+\beta\left(\exp \left(T \alpha t_{2}\right)-\exp \left(T \alpha t_{1}\right)\right)+\exp \left(T \alpha t_{2}\right)-\exp (T \alpha)=-a
$$

It is evident from (44), (45) and (46) that we are able to compute $t_{1}, t_{2}$ and $T$.

\subsection{Control policies for the cascade system}

Let us now proceed to the complete cascade problem. For the sake of simplicity the system (23) to (25) is driven from

to

$$
\left.\begin{array}{l}
x^{\mathrm{T}}(0)=[0, a, 0, \ldots, 0, a] \\
x^{\mathrm{T}}(1)=[0, \ldots, 0]
\end{array}\right\}
$$

in minimal time $T$.

Analogously to (33) and (34), the trajectory is determined by

$$
\begin{aligned}
x_{1}(t) & =T \int_{0}^{t} x_{2}(\tau) d \tau \\
x_{2 j}(t) & =\exp \left(-T \alpha_{j} t\right) T \alpha_{j} \int_{0}^{t} u_{j}(\tau) \exp \left(T \alpha_{j} \tau\right) d \tau+a \exp \left(\begin{array}{r}
\left.-T \alpha_{j} t\right), \\
j=1, \ldots, m
\end{array}\right. \\
x_{2 j-1}(t) & =T \int_{0}^{t} x_{2 j}(\tau) d \tau-T \int_{0}^{t} u_{j-1}(\tau) d \tau, \quad j=2, \ldots, m
\end{aligned}
$$


From (36) we know for $j=1$ with respect to (47)

$$
\int_{0}^{1} u_{1}(\tau) d \tau=\frac{-a}{T \alpha_{1}}
$$

therefore we have

$$
0=x_{3}(1)=T \int_{0}^{1} x_{4}(\tau) d \tau+a / \alpha_{1}
$$

Now we state the new system

$$
\left[\begin{array}{l}
\dot{x}_{3} \\
\dot{x}_{4}
\end{array}\right]=T\left[\begin{array}{cc}
0 & 1 \\
0 & -\alpha_{2}
\end{array}\right]\left[\begin{array}{l}
\tilde{x}_{3} \\
x_{4}
\end{array}\right]+T\left[\begin{array}{l}
0 \\
\alpha_{2}
\end{array}\right] u_{2}
$$

with

$$
\left[\begin{array}{l}
\tilde{x}_{3}(0) \\
x_{4}(0)
\end{array}\right]=\left[\begin{array}{l}
0 \\
a
\end{array}\right], \quad\left[\begin{array}{l}
\tilde{x}_{3}(1) \\
x_{4}(1)
\end{array}\right]=\left[\begin{array}{l}
b_{2} \\
0
\end{array}\right]
$$

where $b_{2}=-a / \alpha_{1}$.

The system (53) resembles (27) and thus it can be solved in the described way. With a solution $\left(u_{2}, T\right)$, we obtain

$$
x_{3}(t)=\tilde{x}_{3}(t)-T \int_{0}^{t} u_{1}(\tau) d \tau
$$

Considering the final condition of $x_{5}$ we have

$$
x_{5}(1)=T \int_{0}^{1} x_{6}(\tau) d \tau-T \int_{0}^{1} u_{3}(\tau) d \tau
$$

With (36) the second term is

$$
T \int_{0}^{1} u_{2}(\tau) d \tau=b_{2}-a / \alpha_{2}
$$

Substituting $b_{2}$ we get for $b_{3}$ as final-condition of $x_{5}$

$$
b_{3}=-a\left(1 / \alpha_{1}+1 / \alpha_{2}\right)
$$

Now we can proceed to the recursive definition of (23) to (25). Set

$$
\left.\begin{array}{rl}
b_{1} & =0 \\
b_{j+1} & :=-a \sum_{i=1}^{j} 1 / \alpha_{i}, j=1, \ldots, m-1
\end{array}\right\}
$$

Introducing subsystems as follows

$$
\begin{gathered}
\left(\mathscr{S}_{j}\right), \quad j=1, \ldots, m \\
{\left[\begin{array}{c}
\dot{x}_{2 j-1} \\
\dot{x}_{2 j}
\end{array}\right]=T^{j}\left[\begin{array}{cc}
0 & 1 \\
0 & -\alpha_{j}
\end{array}\right]\left[\begin{array}{l}
x_{2 j-1} \\
x_{2 j}
\end{array}\right]+T^{j}\left[\begin{array}{l}
0 \\
\alpha_{j}
\end{array}\right] u_{j}}
\end{gathered}
$$


with

$$
\left.\begin{array}{rlrl}
x_{2 j-1}(0) & =0, & x_{2 j-1}(1) & =b_{j} \\
x_{2 j}(0) & =a, & x_{2 j}(1) & =0
\end{array}\right\}
$$

the recursive definition is complete and therefore we are able to solve the entire system (23) to (25) analytically.

If the production rates $x_{2 j}$ are restricted the following constraint holds for each system

$$
S_{j}(x)=x_{2 j}-\beta_{j} \leqq 0
$$

These subsystems with bounded production rates are solvable by means described in $\S 2$.

Let $\left(t_{s}{ }^{j}, T^{j}\right)$ in the case of unbounded subsystems and $\left(t_{1}{ }^{j}, t_{2}{ }^{j}, T^{j}\right)$ for constrained subsystems be the optimal solution of $\left(\mathscr{S}_{j}\right)$. The adjoint equation related to the cascade problem is

$$
\lambda(t)=-A^{\mathrm{T}} \lambda(t)
$$

and as switching function we have

$$
\phi^{\mathrm{T}}(t)=\left(\alpha_{1} \lambda_{2}(t)-\lambda_{3}(t), \alpha_{2} \lambda_{4}(t)-\lambda_{5}(t), \ldots, \alpha_{m} \lambda_{2 m}(t)\right)
$$

Each component $\phi_{j}(t)$ is composed of a constant and an exponential function or zero, respectively. The assumption $\phi_{j}(t) \equiv 0$ for all $j=1, \ldots, m$ contradicts (13). Thus there exists a $j \in\{1, \ldots, m\}$, such that $u_{j}(t)$ is a bang-bang control which governs the subsystem $\left(\mathscr{S}_{j}\right)$. As a consequence the slowest subsystem $\left(\mathscr{S}_{j}\right)$ determines the time $T$ for the entire cascade.

More formally : set $T:=\max \left\{T^{j} \mid j=1, \ldots, m\right\}$. For all subsystems $\left(\mathscr{S}_{j}\right)$ with $T^{j}=T$ determine the bang-bang control $u_{j}$ as described above (see (37), (43)). For all unbounded subsystems with $T^{j}<T$, i.e. which are governed by a singular control we introduce a 'pseudo-bang-bang control ' as follows

$$
u_{j}(t):=\left\{\begin{array}{rr}
1, & 0 \leqq t<t_{s}{ }^{j} \\
-1, & t_{s}{ }^{j} \leqq t \leqq t_{e}{ }^{j} \\
0, & \text { otherwise }
\end{array}\right.
$$

This means that the 'faster' subsystems are controlled as fast as possible by a bang-bang strategy in the first part of the control. In the second part the control is set to zero. Though there are many other possibilities to choose the singular controls it seems to be a reasonable strategy for a minimum time problem.

Thus for all subsystems with $T^{j}<T$ we seek the two switching points $t_{s}^{j}$ and $t_{e}{ }^{j}$. Therefore we have to solve the following equations

$$
\begin{aligned}
2 \exp \left(T \alpha_{j} t_{s}{ }^{j}\right)- & \exp \left(T \alpha_{j} t_{e}{ }^{j}\right)=-a \\
2 T t_{s}{ }^{j}-T t_{e}{ }^{j} & =b_{j}-a / \alpha_{j}
\end{aligned}
$$


Concerning restricted subsystems we seek the entry-point $t_{1}{ }^{j}$, the exit-point $t_{2}{ }^{j}$ and the end-point of the bang-bang control $t_{e}^{j}$ with $0 \leqq t_{1}{ }^{\prime}<t_{2}{ }^{j} \leqq t_{e}{ }^{j} \leqq 1$. With

$$
u_{j}(t):=\left\{\begin{aligned}
1, & 0 \leqq t<t_{1} \\
\beta_{j}, & t_{1} \leqq t \leqq t_{2} \\
-1, & t_{2}<t \leqq t_{c} \\
0, & \text { otherwise }
\end{aligned}\right.
$$

the switching points are obtained by solving the following equations (see (44) (45), (46))

$$
\begin{gathered}
t_{1}{ }^{j}=\frac{\ln \frac{1-a}{1-\beta_{j}}}{T \alpha_{j}} \\
t_{1}{ }^{j}+\beta_{j}\left(t_{2}{ }^{j}-t_{1}{ }^{j}\right)+t_{2}{ }^{j}-t_{e}{ }^{j}=\frac{b_{j}}{T}-\frac{a}{T \alpha_{j}} \\
\exp \left(T \alpha_{j} t_{1}{ }^{j}\right)-1+\beta_{j}\left(\exp \left(T \alpha_{j} t_{2}{ }^{j}\right)-\exp \left(T \alpha_{j} t_{1}{ }^{j}\right)\right) \\
+\exp \left(T \alpha_{\alpha} t_{2}{ }^{j}\right)-\exp \left(T \alpha_{j} t_{e}{ }^{j}\right)=-a
\end{gathered}
$$

An important property of the chosen 'pseudo-bang-bang control' is the fact that there is a continuous transition between singular and bang-bang controls. If the constraints of the singular controlled subsystems are strengthened, $t_{e}^{j}$ approaches one. At the point $t_{e}^{j}=1$ we have $T^{j}=T$ and the regarded subsystem becomes a bang-bang controlled one. If the constraint is strengthened further the subsystem remains bang-bang controlled and all other subsystems will be pseudo-bang-bang controlled.

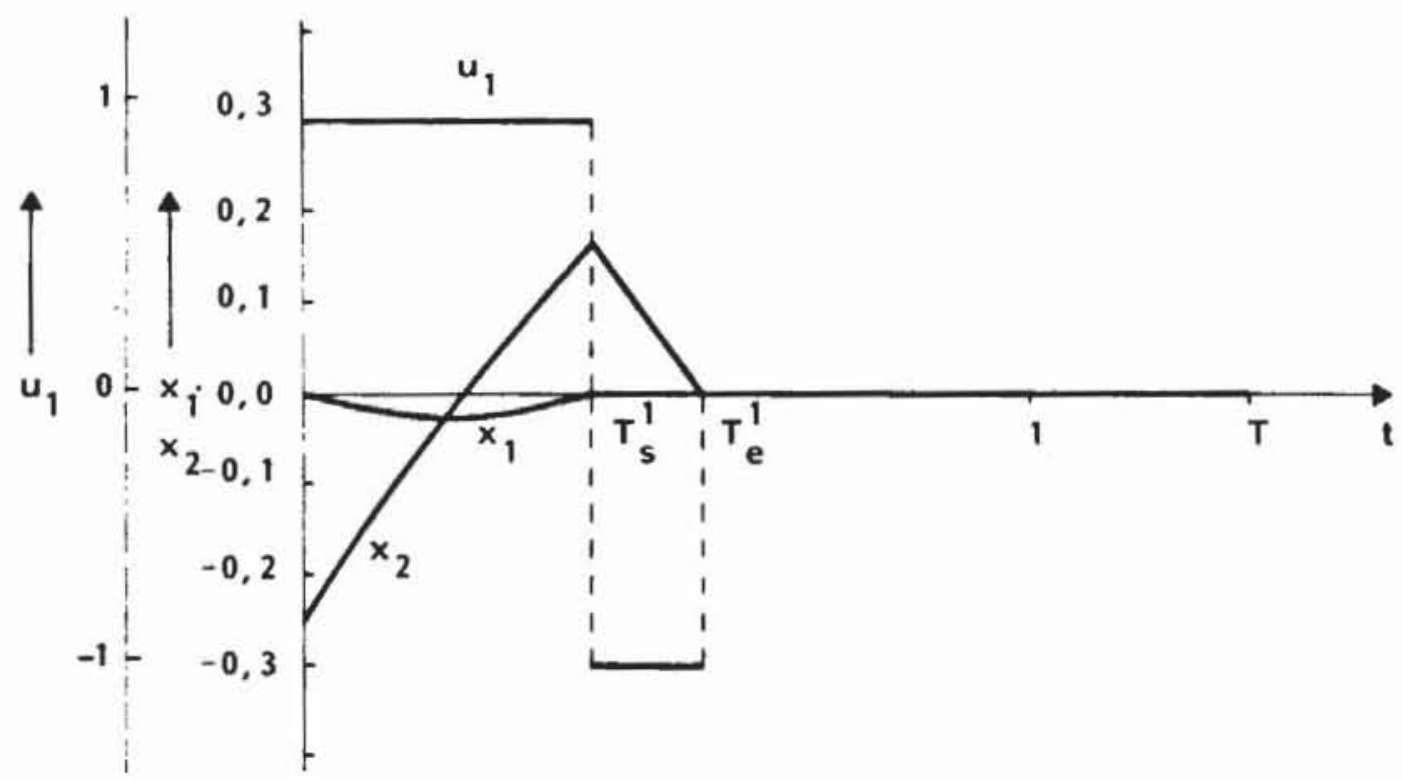

Figure 2. 


\section{Remark}

If all production rates are unbounded the last subsystem $\mathscr{S}_{m}$ is bang-bang and the other systems are singular controlled.

\subsection{Numerical example}

As illustration of the theory presented in $\S 3.3$ we consider a system consisting of two subsystems. For the ease of demonstration the parameters are assumed as follows : $\alpha_{1}=\alpha_{2}=1, s_{0}=-0 \cdot 25, s_{T}=0 \cdot 0$. The time scale is let unnormed. Figures (2) and (3) show the history of the optimal control and state variables for the subsystems 1 and 2 , respectively, in the unconstrained

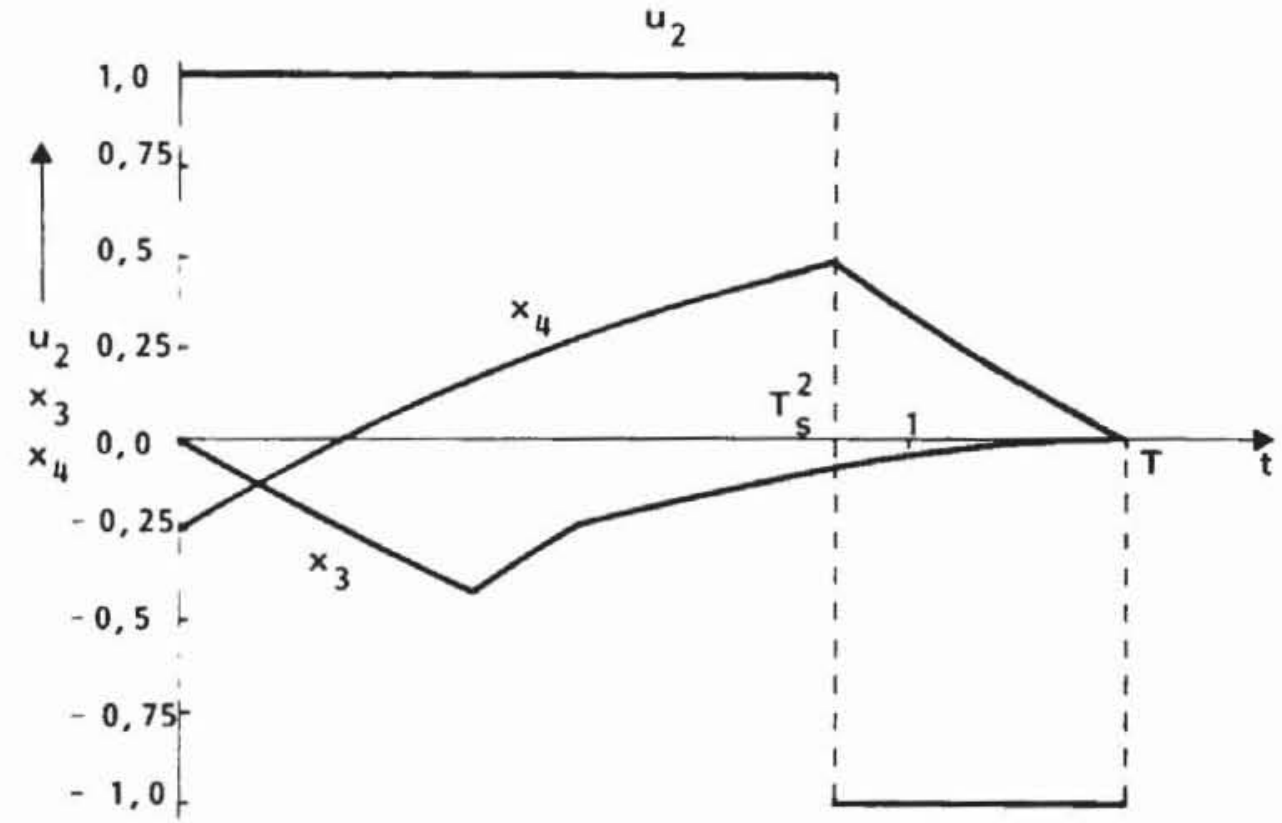

Figure 3.

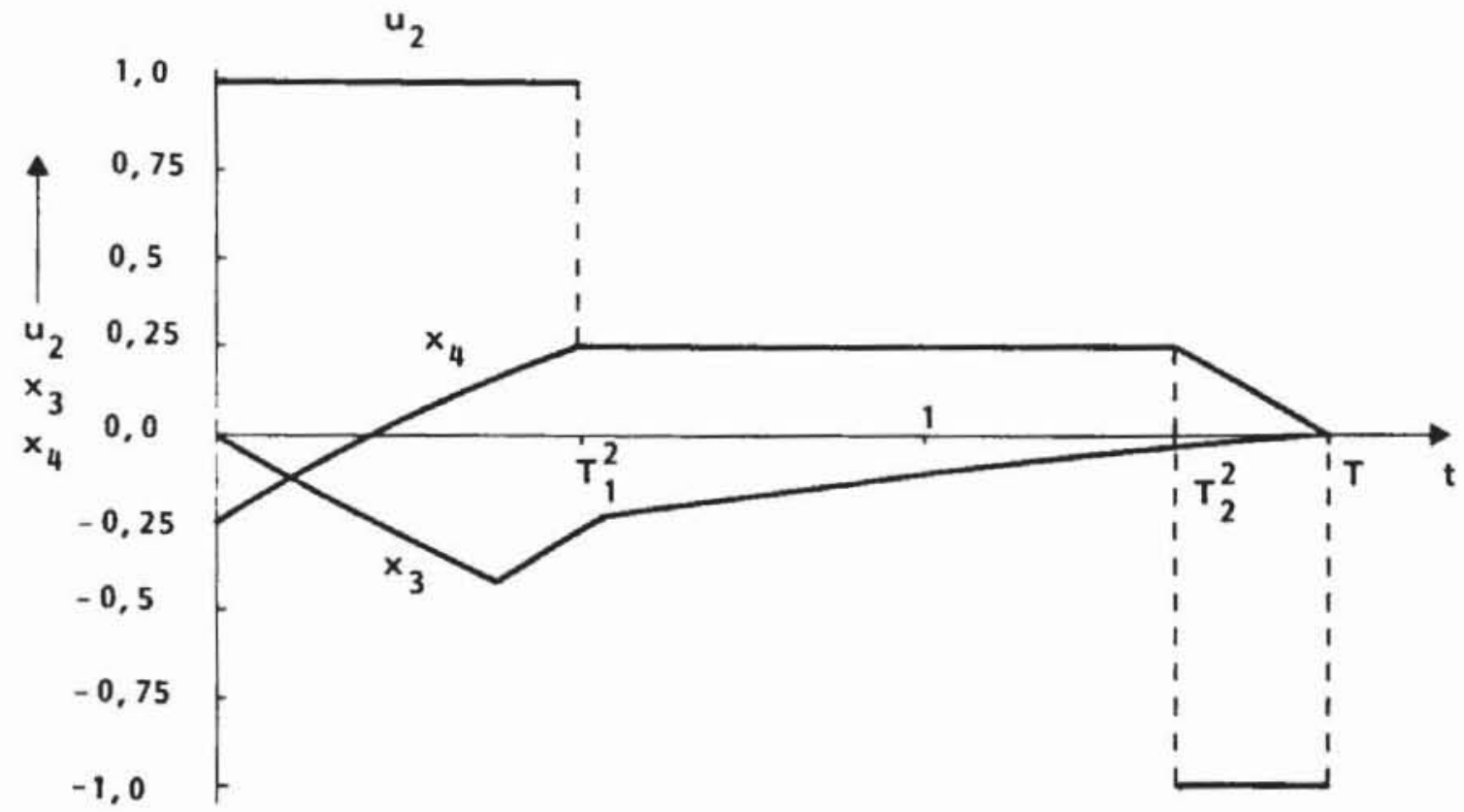

Figure 4. 
case. The minimum time period is $T=1 \cdot 2999$, the switching times for the first subsystem are $T_{*}{ }^{1}=0.4008$ and $T_{e}{ }^{1}=0.5516$. For the second subsystem the switching time is calculated as $T_{n}{ }^{2}=0 \cdot 8999$. Figure (t) illustrates the constrained case. Assuming for the second production-inventory system a maximum production rate $\beta_{2}=0 \cdot 25$ which cannot be exceeded the state constraint takes the form $S_{2}(x(t))=x_{4}(t)-0 \cdot 25 \leqq 0$. The trajectories of the first subsystem remain exactly the same as in the unconstrained case, excepted the minimal time period which becomes $T=1.5832$. The variable $x_{4}(t)$ of the second subsystem lies on the boundary between the entry time $T_{1}{ }^{2}=0.5108$ and the exit time $T_{2}^{2}=1 \cdot 3601$.

In this paper time-optimal control policies have been derived for cascaded production-inventory systems with control and state constraints. It has been shown that the problem can be solved analytically due to a recursive definition of the cascaded system by means of the minimum principle for state constrained systems. It has been pointed out that at least one subsystem is bang-bang controlled whereas the others are singular controlled. By introducing a 'pseudo-bang-bang control' the transition from bang-bang control to singular control and vice versa has been realized particularly for constrained problems.

The theory has been illustrated by the presentation of calculated results concerning two production-inventory subsystems in cascade for the unconstrained and the constrained case.

\section{REFERENCES}

Bradshaw; A., and Erol, Y., 1980, Int. J. Systems Sci., 11, 947.

Grasaxov. I. Y.. 1972, Lectures on Mathematical Theory of Extremum Problems (Berlin : Springer).

Haumton, W. E., 1972, I.E.E.E. Trans. autom. Control, 17, 338.

JACobson, D. H., and Lele, M. M., and SPEYer, J. L., 1971, J. math. Analysis Applic., 35, 255.

MLURER, H., 1977, SI A M J. Control, 15, 345. 